\section{Sustained Elevation in Monocyte Levels in Diabetic Patients after Infra- Inguinal Revascularization}

\section{Abstract}

Context: Diabetes and atherosclerosis are both pro-inflammatory states that may lead to elevations in monocyte levels. It was previously demonstrated that there is a reduction in monocyte levels after infra-inguinal bypass in patients with critical limb ischemia (CLI). We hypothesized that patients with diabetes would not realize the same reduction in monocyte levels post-bypass as patients without diabetes.

Objective: To determine whether patients with diabetes would have a sustained sub-clinical inflammation after resolution of CLI with infra-inguinal bypass, as marked by a persistent elevation of monocyte levels.

Design: Patients undergoing lower extremity vascular bypass surgery between 2003 and 2013 at the Syracuse VA Medical Center were retrospectively reviewed. Pre- and post-operative leukocyte count with differential were recorded for each patient and stratified according to the presence of diabetes.

Results: Patients with $\mathrm{CLI}$ and no bypass failure $(n=43)$ were included for analysis of the primary outcome, change in monocyte level. Diabetic $(D M+)$ patients $(n=27)$ and non-diabetic (DM-) patients $(n=16)$ had similar pre-operative leukocyte counts and differential ( $p>0.05$ ). In DM-, there was a 5.6\% decrease in monocyte count post-operatively, whereas there was an elevation in monocytes in $\mathrm{DM}+(+20.8 \%$; $\mathrm{p}<0.05)$. The overall rate of complications was significantly greater and the time to develop complications significantly less in $\mathrm{DM}+(\mathrm{p}<0.05)$.

Conclusion: Diabetic patients have a persistent elevation in monocyte levels even after infra-inguinal vascular bypass as compared with non-diabetic patients. This suggests diabetic patients have persistent sub-clinical inflammation even upon resolution of critical limb ischemia.

Keywords: Infra-inguinal bypass; Monocyte; Diabetes; Smoking

Abbreviations: CLI: Critical Limb Ischemia; DM+: Patients with Diabetes; DM-: Patients without Diabetes; PAD: Peripheral Arterial Disease

\section{Michaela \\ Kollisch Singule $e^{1,2}$, Benjamin Sadowitz ${ }^{3}$, Qinghe Meng ${ }^{1}$, Michael J Costanza ${ }^{1,2}$ and Kwame Amankwah ${ }^{1,2}$}

\section{Department of Surgery, SUNY Upstate Medical University, Syracuse, NY, USA \\ 2 Department of Surgery, Syracuse VAMC, Syracuse, NY, USA \\ 3 Department of Surgery, Crouse Hospital, Syracuse NY, USA}

\section{Corresponding author: \\ Kwame Amankwah}

ఏAmankwaK@upstate.edu

SUNY Upstate Medical University, $750 \mathrm{E}$ Adams Street Syracuse, NY 13210,USA.

Tel: (315) 4646241

Citation: Singule MK, Sadowitz B, Meng $Q$, et al. Sustained Elevation in Monocyte Levels in Diabetic Patients after InfraInguinal Revascularization Journal of Vascular \& Endo Surgery. 2016, 1:1.

\section{Introduction}

The role of monocytes on the development of peripheral arterial disease (PAD) and critical limb ischemia (CLI) has been a source of great interest given the potential for therapeutic opportunities $[1,2]$. It has previously been demonstrated that monocyte levels decrease after revascularization [2], consistent with the fact that they play an integral role in the early stages of atherosclerosis initiation, plaque rupture, and remodeling [3, 4]. The presence of diabetes potentiates this process through a combination of increasing oxidative stress [5] and non-enzymatic glycation of molecules which activate inflammatory mediators and macrophages and encourage atheroma formation [3]. The endothelial cells of diabetics secrete cytokines that inhibit the synthesis of new collagen by vascular smooth muscle cells and promote the production of matrix metalloproteinases, allowing plaques to rupture more easily and form thrombi [5]. Over time, this process leads to occlusion of the vessel, decreased arterial perfusion, and ultimately $\mathrm{CLI}$ and limb loss. Diabetes induces 
a pro-inflammatory state with a tendency toward monocyte recruitment and activation, suggesting that patients with diabetes may have ongoing sub - clinical inflammation as measured by monocyte elevation.

In this study, we investigated monocyte levels before and after surgical revascularization in patients with CLI. Specifically, we hypothesized that patients with diabetes would have a sustained elevation of monocyte levels after vascular bypass procedures as compared with patients without diabetes. This preliminary retrospective study demonstrates that monocyte levels do not necessarily decrease after revascularization in patients with diabetes. This serves as a foundation for future prospective studies investigating monocyte levels in patients with and without diabetes after bypass, including measuring the ratio of pro-inflammatory to anti-inflammatory monocytes, as a potential monitoring tool.

\section{Methods}

All patients who underwent a lower extremity bypass surgery between 2003 and 2013 at the Syracuse VA Medical Center were reviewed retrospectively. Patients who underwent inflow procedures including femoral-femoral bypass or aortobifemoral/ axillofemoral bypasses were excluded from this study. Leukocyte levels with differentials including neutrophils, lymphocytes, monocytes, eosinophils and basophils were recorded at two time points: pre-operative and post-operative. The pre-operative time point closest to the day of the procedure was recorded and post-operative leukocyte counts nearest to one year postoperatively were preferentially selected, however there was a wide time range, consistent with the nature of a retrospective study (Figure 1). Patients were excluded from the study if they did not have a leukocyte count with differential for both data points. Patients requiring more than one bypass during the study period were included only once for analysis, but only the data from their most recent bypass was analyzed. If the pre-operative leukocyte count was within one year of a previous bypass, the patient was excluded from the study. For each cell count within the differential, the percent change between pre- and postoperative values was calculated.

Patients were classified according to Fontaine Stage in order to stratify whether or not they had CLI. Patients with Fontaine stage III (rest pain) or IV (ischemic ulcers or gangrene) were classified as having $\mathrm{CLI}$ [6]. Since the primary objective of the study was to analyze the response of monocyte levels in diabetic patients with $\mathrm{CLI}$ after bypass, patients with Fontaine stage I (asymptomatic disease) or II (intermittent claudication) were excluded from the study [6]. Patients with a failed graft, as defined by one of: post-operative graft occlusion, classification as Fontaine stage III or IV post-operatively, or limb loss were excluded from the study so as not to include secondarily elevated monocyte levels. Patients were classified as diabetic $\left(\mathrm{DM}^{+}\right)$if they (a) had a current diagnosis of diabetes at the time of the surgery, (b) regularly took an anti-hyperglycemic agent or (c) had a hemoglobin A1C (HgbA1c) level greater than $6.5 \%$. Patients who did not meet any of these criteria were classified as non-diabetic $\left(\mathrm{DM}^{-}\right)$.

Patient factors including demographics, co-morbidities, surgical variables, medications and pre-operative lab values were also elicited by chart review. Patient demographics included: age at time of surgery, race, and gender. Co-morbidities included: smoking status, body mass index (BMI), presence of heart disease, hypertension, renal disease and chronic obstructive pulmonary disease (COPD). Surgical variables included: side of bypass, type of graft, and inflow and outflow vessel. Medications analyzed included: statins, ACE-inhibitors, beta-blockers, calcium channel blockers and antiplatelet/anticoagulation therapy. Pre-operative lab values included: leukocyte counts with differential, albumin, serum creatinine and HgbA1c. Outcome measures including pre- and post-operative ankle-brachial indices (ABI), myocardial infarction, new arrhythmia, stroke, minor infections, cellulitis, osteomyelitis, ulceration or gangrene of the revascularized limb, graft status, limb status and mortality were also recorded. Mean follow-up time and time to death were also recorded.

As mentioned previously, patients who developed bypass failure,

\section{Number of Days CBC with Differential Preceded Bypass by Frequency of Patients}

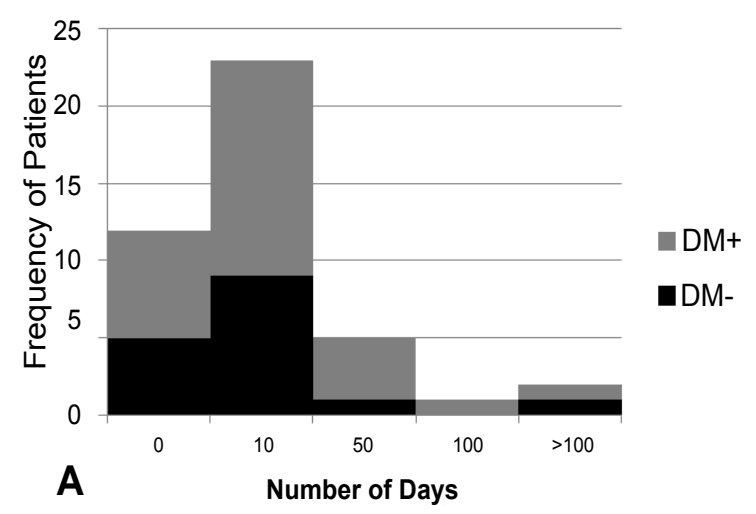

Number of Days CBC with Differential Succeeded Bypass by Frequency of Patients

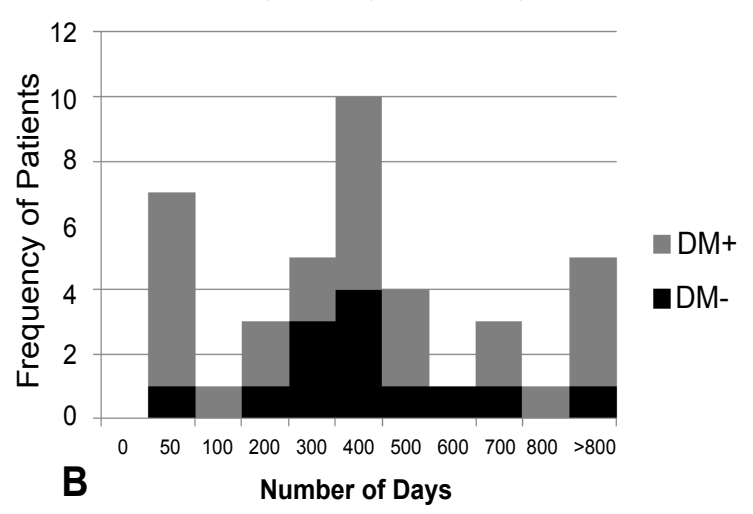


as defined by limb loss, graft occlusion, or inability to repair the graft with one endovascular intervention, were excluded from the primary analysis. Limb loss was defined as the requirement of a major amputation (below-knee or above-knee amputation) but patients requiring minor amputations (toe or transmetatarsal amputation) were included in the study. In order to demonstrate cumulative survival after bypass, overall graft longevity and limb salvage, all patients undergoing a bypass for $\mathrm{CLI}$, including those with bypass failure, were included in a Kaplan-Meier analysis.

\section{Ethics}

The study was approved by the Institutional Review Board at the Syracuse VA Medical Center, Syracuse, NY.

\section{Statistics}

Statistical analyses were conducted with SPSS 11.5 (SPSS Inc., Chicago IL). Categorical variables were analyzed using univariate analysis and continuous variables were analyzed using ANOVA. A multivariate general linear model was used to determine percent change in monocyte levels associated with diabetes and adjust for possible confounding factors (age, body mass index, hypertension, heart disease, renal disease, chronic obstructive pulmonary disease, pre-operative medications and surgical variables). Survival, graft longevity, limb salvage and complication rates were analyzed using Kaplan-Meier statistics and curves were compared with the log rank test. Results are reported as mean \pm SEM and $p$-values $<0.05$ were considered statisticallysignificant.

\section{Results}

\section{Demographics}

A total of 70 lower extremity peripheral bypass surgeries were performed at the Syracuse VA between January 2003 and December 2013 (Figure 2). In this group, 73\% patients ( $n=61$ ) had critical limb ischemia (CLI; Fontaine Stage III-IV) and the remaining $27 \%(n=9)$ were classified as Fontaine Stage I-II, being treated for asymptomatic popliteal aneurysms or intermittent claudication unrelieved by conservative measurements since Fontaine Stage I-II is not conventionally treated by a surgical approach [7]. Patients without both a pre- and post-operative monocyte level $(n=3)$ were excluded as well as those who developed bypass failure $(n=15)$. After applying the inclusion and exclusion criteria, 43 patients remained as the primary focus of this study.

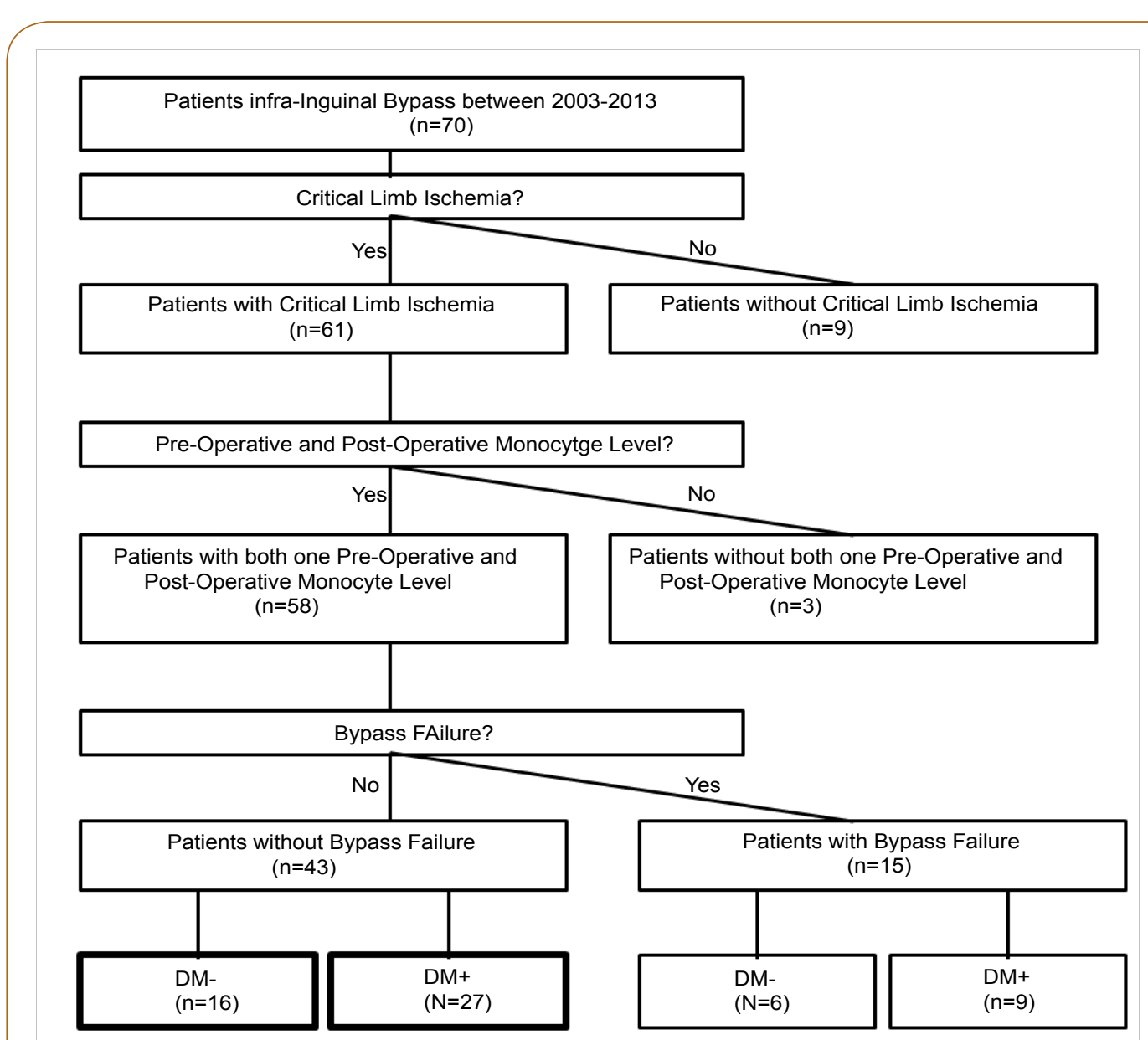

Figure 2 Inclusion and exclusion criteria for patients in the study. Patients requiring in-flow procedures were not considered for the study. Patients without critical limb ischemia (Fontaine Stage I or II) and those without a pre-and post-operative monocyte level were excluded. Patients with bypass failure as defined by post-operative graft occlusion, re-classification as Fontaine stage III or IV postoperatively, or limb loss were not included in analysis of the primary outcome. $\mathrm{DM}^{-}=$patients without diabetes. $\mathrm{DM}^{+}=$patients with diabetes. 
Of these 43 patients, $63 \%$ had diabetes $\left(\mathrm{DM}^{+}: \mathrm{n}=27\right)$ and $37 \%$ did not $\left(D^{-}: n=16\right)$. The complete demographics of these two groups are listed in Table 1. Eleven of the $16 \mathrm{DM}^{-}$patients $(69 \%)$ and 14 of the $38 \mathrm{DM}^{+}$patients $(52 \%)$ were classified as Fontaine Stage III pre-operatively $(p>0.05)$ with the remainder in each group categorized as Fontaine Stage IV ( $D M=n=5 ; M^{+}$: $\mathrm{n}=13) . \mathrm{DM}^{+}$had a similar pre - operative $\mathrm{ABI}(0.46 \pm 0.04)$ as

Table 1 Demographics of patients with diabetes $\left(\mathrm{DM}^{+}\right)$and without diabetes $\left(\mathrm{DM}^{-}\right)$with critical limb ischemia who underwent infra-inguinal revascularization without graft failure. ${ }^{*}=p<0.05$.

\begin{tabular}{|c|c|c|c|}
\hline Demographics & $\mathrm{DM}^{-}(\mathrm{n}=16)$ & $\mathrm{DM}^{+}(\mathrm{n}=27)$ & P-value \\
\hline \multicolumn{4}{|c|}{ Sex } \\
\hline Male & $16(100 \%)$ & 27 (100\%) & $>0.9999$ \\
\hline Female & $0(0 \%)$ & $0(0 \%)$ & \\
\hline \multicolumn{4}{|c|}{ Race } \\
\hline Caucasian & $15(94 \%)$ & $26(96 \%)$ & \\
\hline African American & $1(6 \%)$ & $0(0 \%)$ & 0.3187 \\
\hline Other & $0(0 \%)$ & $1(4 \%)$ & \\
\hline Age & $62.8 \pm 2.23$ & $70.5 \pm 1.82$ & $0.0122 *$ \\
\hline BMI & $26.28 \pm 1.07$ & $29.2 \pm 0.887$ & $0.0458^{*}$ \\
\hline \multicolumn{4}{|c|}{ Smoker } \\
\hline Current & $14(87 \%)$ & $11(41 \%)$ & \\
\hline Prior & $2(13 \%)$ & 15 (55\%) & $0.0107^{*}$ \\
\hline Never & $0(0 \%)$ & $1(4 \%)$ & \\
\hline \multicolumn{4}{|l|}{ Fontaine Stage } \\
\hline Stage III & $11(69 \%)$ & $14(52 \%)$ & \multirow{2}{*}{0.2736} \\
\hline Stage IV & $5(31 \%)$ & $13(48 \%)$ & \\
\hline Pre-Operative $\mathrm{ABI}$ & $0.51 \pm 0.05$ & $0.46 \pm 0.04$ & 0.4396 \\
\hline Post-Operative ABI & $0.91 \pm 0.04$ & $0.97 \pm 0.04$ & 0.2888 \\
\hline Change in $\mathrm{ABI}$ & $0.40 \pm 0.06$ & $0.51 \pm 0.03$ & 0.0926 \\
\hline \multicolumn{4}{|l|}{ Patient Co-Morbidities } \\
\hline \multicolumn{4}{|l|}{ Heart Disease } \\
\hline Yes & $9(56 \%)$ & $9(33 \%)$ & 0.1409 \\
\hline No & $7(44 \%)$ & $18(67 \%)$ & \\
\hline \multicolumn{4}{|l|}{ Hypertension } \\
\hline Yes & $12(75 \%)$ & $26(96 \%)$ & $0.0352 *$ \\
\hline No & $4(25 \%)$ & $1(4 \%)$ & \\
\hline \multicolumn{4}{|l|}{ Renal Disease } \\
\hline Yes & $0(0 \%)$ & $9(33 \%)$ & \\
\hline No & $16(100 \%)$ & $18(67 \%)$ & $0.0094^{*}$ \\
\hline \multicolumn{4}{|c|}{ Chronic Obstructive Pulmonary Disease } \\
\hline Yes & $7(44 \%)$ & $8(30 \%)$ & 0.3477 \\
\hline No & $9(56 \%)$ & $19(70 \%)$ & \\
\hline \multicolumn{4}{|l|}{ Statin } \\
\hline Yes & $7(44 \%)$ & 20 (74\%) & $0.0468^{*}$ \\
\hline No & $9(56 \%)$ & $7(26 \%)$ & \\
\hline \multicolumn{4}{|l|}{ ACE-Inhibitor } \\
\hline Yes & $6(38 \%)$ & $14(52 \%)$ & 0.3618 \\
\hline No & $10(62 \%)$ & $13(48 \%)$ & \\
\hline \multicolumn{4}{|l|}{ Beta-Blocker } \\
\hline Yes & $6(38 \%)$ & $20(74 \%)$ & $0.0177^{*}$ \\
\hline No & $10(62 \%)$ & $7(26 \%)$ & \\
\hline \multicolumn{4}{|l|}{ Calcium Channel Blocker } \\
\hline Yes & $3(19 \%)$ & $7(26 \%)$ & 0.5903 \\
\hline No & $13(81 \%)$ & $20(74 \%)$ & \\
\hline
\end{tabular}

\begin{tabular}{|c|c|c|c|}
\hline \multicolumn{4}{|c|}{ Anti-Platelet Prior to Surgery } \\
\hline Yes & $6(38 \%)$ & $16(59 \%)$ & 0.1677 \\
\hline No & $10(62 \%)$ & $11(41 \%)$ & \\
\hline \multicolumn{4}{|l|}{ Anti-Platelet Post-Surgery } \\
\hline Yes & $12(75 \%)$ & $23(85 \%)$ & 0.4127 \\
\hline No & $4(25 \%)$ & $4(15 \%)$ & \\
\hline \multicolumn{4}{|l|}{ Anti-Coagulation } \\
\hline Yes & $6(38 \%)$ & $7(26 \%)$ & 0.4244 \\
\hline No & $10(62 \%)$ & $20(74 \%)$ & \\
\hline \multicolumn{4}{|l|}{ Surgical Variables } \\
\hline \multicolumn{4}{|l|}{ Graft Type } \\
\hline Native Vein & $13(81 \%)$ & $20(74 \%)$ & 0.5903 \\
\hline Prosthetic & 3 (19\%) & $7(26 \%)$ & \\
\hline \multicolumn{4}{|l|}{ Outflow Vessel } \\
\hline Above Knee Popliteal & $8(50 \%)$ & $10(37 \%)$ & \multirow{5}{*}{0.1625} \\
\hline Below Knee Popliteal & $0(0 \%)$ & $2(7 \%)$ & \\
\hline Posterior Tibialis & $6(38 \%)$ & $4(15 \%)$ & \\
\hline Anterior Tibialis & $1(6 \%)$ & $7(26 \%)$ & \\
\hline Peroneal & $1(6 \%)$ & $4(15 \%)$ & \\
\hline \multicolumn{4}{|l|}{ Extremity } \\
\hline Right & $9(56 \%)$ & $16(59 \%)$ & 0.8467 \\
\hline Left & $7(44 \%)$ & $11(41 \%)$ & \\
\hline \multicolumn{4}{|l|}{ Pre - Operative Lab Values } \\
\hline Albumin (g/dL) & $3.48 \pm 0.15$ & $3.03 \pm 0.13$ & $0.0324 *$ \\
\hline Creatinine (mg/dL) & $0.91 \pm 0.07$ & $1.24 \pm 0.15$ & 0.1270 \\
\hline $\operatorname{HgbA1c}(\%)$ & $5.47 \pm 0.20$ & $7.79 \pm 0.32$ & $<0.0001^{*}$ \\
\hline Leukocytes (× 109/L) & $9.23 \pm 0.71$ & $8.19 \pm 0.37$ & 0.1621 \\
\hline Neutrophils $\left(\times 10^{9} / \mathrm{L}\right)$ & $6.36 \pm 0.61$ & $5.77 \pm 0.38$ & 0.3987 \\
\hline Lymphocytes (× 10\%/L) & $1.79 \pm 0.20$ & $1.48 \pm 0.10$ & 0.1325 \\
\hline Monocytes (× 109/L) & $0.76 \pm 0.06$ & $0.69 \pm 0.05$ & 0.3514 \\
\hline Eosinophils $\left(\times 10^{9} / \mathrm{L}\right)$ & $0.26 \pm 0.06$ & $0.23 \pm 0.07$ & 0.6304 \\
\hline Basophils $\left(\times 10^{9} / \mathrm{L}\right)$ & $0.02 \pm 0.01$ & $0.02 \pm 0.01$ & 0.9854 \\
\hline
\end{tabular}

compared with $\mathrm{DM}^{-}(0.51 \pm 0.05 ; \mathrm{p}>0.05)$ and a similar increase post - operatively $(p>0.05) . \mathrm{DM}^{+}$had a lower albumin $(p<0.05)$ and higher HgbA1c $(p<0.05)$ as compared with $\mathrm{DM}^{-}$. The average age of surgery was $70.5 \pm 1.82 \mathrm{y}$ in $\mathrm{DM}^{+}$as compared with 62.8 $\pm 2.23 \mathrm{y}$ in $\mathrm{DM}^{-}$patients $(\mathrm{p}<0.05)$. $\mathrm{DM}^{+}$also tended to have a higher BMI $(p<0.05)$ but a lower incidence of current smoking $(\mathrm{p}<0.05)$ as compared with $\mathrm{DM}^{-}$. $\mathrm{DM}^{+}$had a higher incidence of renal disease, hypertension, beta-blocker use and statin use as compared with $\mathrm{DM}^{-}(\mathrm{p}<0.05)$. There was no significant difference between the two groups with regards to sex, race, prevalence of heart disease or COPD, graft type, outflow vessel, or extremity.

After adjusting for age, BMI, presence of heart disease or COPD, use of antiplatelet agents, ACE - Inhibitors or beta-blockers, type of graft, side of graft, whether or not the bypass was a revision, and smoking status (never/prior versus current), diabetes remained a significant determinant of post-operative monocyte level ( $p<0.05 ;$ Table 2).

\section{Leukocyte count and differential}

Pre-operative leukocyte count and differential were similar in both groups of patients (Table 1). $\mathrm{DM}^{-}$had a post-operative reduction in leukocytes as compared with $\mathrm{DM}^{+}$although it was not statistically significant $(-0.1 \%$ vs. $+3.7 \%$; $p>0.05$; Figure $3 A)$. 
Both $\mathrm{DM}^{-}$and $\mathrm{DM}^{+}$had a small increase in neutrophils $(5.1 \%$ vs. 9.0\%; $p>0.05$; Figure 3B) and lymphocytes (+3.3\% vs. $+12.8 \%$; $\mathrm{p}>0.05$; Figure $3 \mathrm{C}$ ) post-operatively. $\mathrm{DM}^{-}$patients had a postoperative reduction of eosinophils $(-20.1 \%)$ as compared with $\mathrm{DM}^{+}(+11.4 \%$; $>0.05$; results not shown). The basophil count ranged between 0.0 and 0.2 in both $\mathrm{DM}^{+}$and $\mathrm{DM}^{-}$in the pre - and post-operative period. Because of a significant number of basophil values of 0.0 pre-operatively, a percent reduction could not be calculated due to the " 0 " in the denominator.

After bypass, there was a relative reduction in monocyte count in the $\mathrm{DM}^{-}$group from $0.76 \pm 0.06 \times 10^{9}$ cells/L pre-operatively to $0.69 \pm 0.06 \times 10^{9}$ cells/L post - operatively. By comparison, the post-operative monocyte count in $\mathrm{DM}^{+}\left(0.76 \pm 0.06 \times 10^{9}\right.$ cells/L) was relatively greater than the pre-operative monocyte count $\left(0.69 \pm 0.05 \times 10^{9}\right.$ cells/L). The average percent change in monocyte count between pre- and post-bypass was decreased by

Table 2 Association between percent change in monocytes and diabetes unadjusted and adjusted for age, body mass index, presence of heart disease, chronic obstructive pulmonary disease and renal disease, use of antiplatelet agents, ACE-Inhibitors and beta-blockers, type of graft, side of graft, whether or not the bypass was a revision and smoking status (never/prior versus current).

\begin{tabular}{|c|c|c|c|}
\hline & $\mathrm{B}$ & $\mathrm{T}$ & $\mathrm{P}$ Value \\
\hline Unadjusted & -0.264 & 2.063 & 0.045 \\
\hline Adjusted* & -0.41 & 2.063 & 0.048 \\
\hline
\end{tabular}

$5.6 \%$ in the $\mathrm{DM}^{-}$group as compared with a $20.8 \%$ increase in the $\mathrm{DM}^{+}$group ( $p<0.05$; Figure 3D).

\section{Outcomes}

Although complications occurred more frequently and over a shorter time span in $\mathrm{DM}^{+}\left(p<0.05\right.$; Figure 4A), $\mathrm{DM}^{+}$and $\mathrm{DM}^{-}$ had similar survival rates (Figure 4B). Patients without diabetes had increased rates of persistent claudication as compared with $\mathrm{DM}^{+}$post-operatively ( $\mathrm{p}<0.05$; Table 3 ). $\mathrm{DM}^{+}$had a trend towards higher rates of toe amputations and transmetatarsal amputations (44\%) as compared with $\mathrm{DM}^{-}(19 \%)$ but this was not statistically significant $(p=0.09)$. Two $\mathrm{DM}^{+}$patients required a split-thickness skin graft for non-healing wounds. There was no statistically significant difference in infection such as cellulitis or osteomyelitis between the two groups despite the differences in the percent change in monocyte levels.

Six of the $22 \mathrm{DM}^{-}$patients with $\mathrm{CLI}(27 \%)$ and 9 of the $36 \mathrm{DM}^{+}$ patients with $\mathrm{CLI}(25 \%)$ were eliminated due to graft failure, as this would confound the interpretation of whether a persistent monocyte elevation was due to subclinical inflammation from diabetes versus a monocyte elevation secondary to an unperfused limb. In order to determine the rates of cumulative limb salvage and graft patency, these 15 patients who developed bypass failure were added to the 43 patients that were the primary focus in this study. The cumulative limb salvage rate and graft patency in these 58 patients was similar (Figures $4 \mathrm{C}$ and

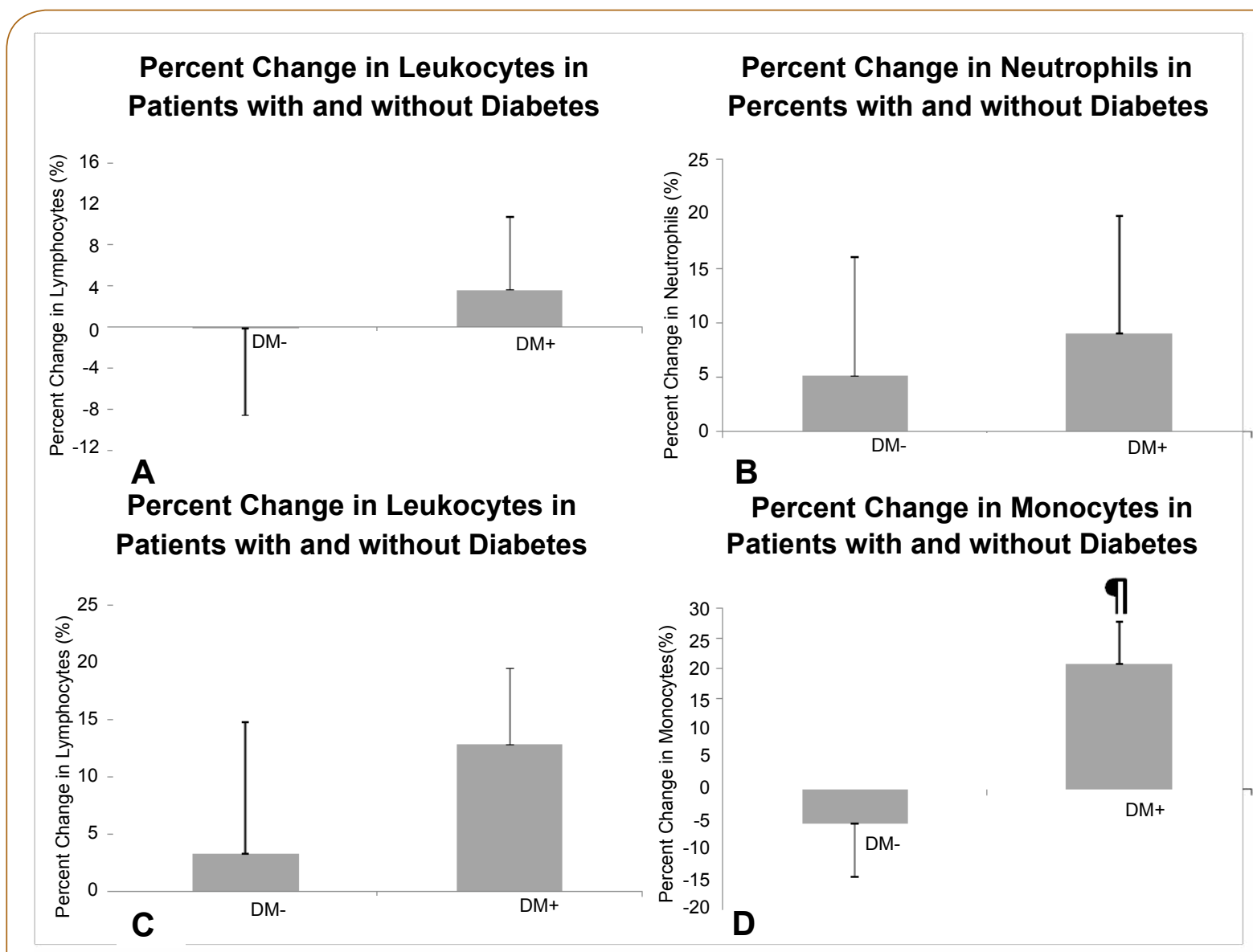

Figure 3

Percent change in leukocyte differential pre- and post-lower extremity bypass in patients with diabetes $\left(\mathrm{DM}^{+}\right)$and without diabetes $\left(\mathrm{DM}^{-}\right)$. Depicted are the percent changes in leukocytes (A), neutrophils (B), lymphocytes (C) and monocytes (D). 
4D), but the time to develop graft failure between the groups differed. $\mathrm{DM}^{-}$developed graft failure an average of $915 \pm 285$ days post-operatively whereas $\mathrm{DM}^{+}$developed graft failure an average of $240 \pm 97$ days post-operatively ( $p<0.05$ ). $\mathrm{DM}^{-}$patients who developed graft failure were all current smokers at the time of the bypass, whereas only 4 of 9 of the $\mathrm{DM}^{+}$patients who developed graft failure were current smokers (Figure 4).

\section{Discussion}

We found that diabetic patients with CLI had elevated monocyte levels after infra-inguinal vascular bypass as compared with nondiabetic patients. Although graft failure and limb salvage rates were similar between diabetic and non-diabetic patients, the increased prevalence of active smoking in non-diabetic patients may explain this result.

In a previous retrospective study of 32 patients undergoing lower extremity bypass surgery, preoperative monocyte levels were elevated in patients with $\mathrm{CLI}$ as compared with those without $\mathrm{CLI}$, and post-operative monocyte levels significantly decreased [2]. The authors of this study speculated that the preoperative monocyte elevation reflected the role of monocytes in angiogenesis while the post-operative monocyte reduction indicated relief of limb ischemia [2]. In that study [2] patients with diabetes had significantly reduced postoperative monocyte counts compared to non-diabetic patients however diabetes did not predict diminished monocyte count on multivariable logistic regression. In contrast to these findings, we hypothesized that diabetics with CLI would have elevated monocyte levels even after revascularization because diabetes is a pro-thrombogenic, pro-inflammatory, and pro-oxidative state [8].

In the context of critical limb ischemia, inflammatory cells, including circulating monocytes, are recruited to the site of endothelial injury [9]. They are integral both to the initiation of the plaque as well as to plaque rupture [10]. Activated monocytes express the CD40-CD40 ligand, which plays a role in inflammatory and pro-thrombotic processes due to release of inflammatory cytokines, chemokines, adhesion molecules and tissue factor [11, 12]. As compared with age- and sex-matched patients without diabetes, patients with diabetes have an increase in plateletmonocyte aggregates and soluble CD40L $[12,13]$. These results suggest that the pro-inflammatory and pro-thrombotic influence of diabetes predisposes diabetic patients to PAD and subsequent CLI [12].

In order to characterize changes in monocyte activation in patients with $\mathrm{CLI}$ but also to determine the effect of surgical stress on monocyte activation, an observational, cross-sectional study was performed comparing patients with $\mathrm{CLI}$ with patients with chronic intermittent claudication, patients with non-ST segment elevation, healthy patients undergoing hip or knee arthroplasty, and healthy volunteers [10]. In that study [10] blood samples were taken from patients the day before operation, immediately post-operatively, and the day after the operation. Monocyte expression of CD40 was greatest in patients with $\mathrm{CLI}$ at baseline and monocyte CD11b was greatest in patients with
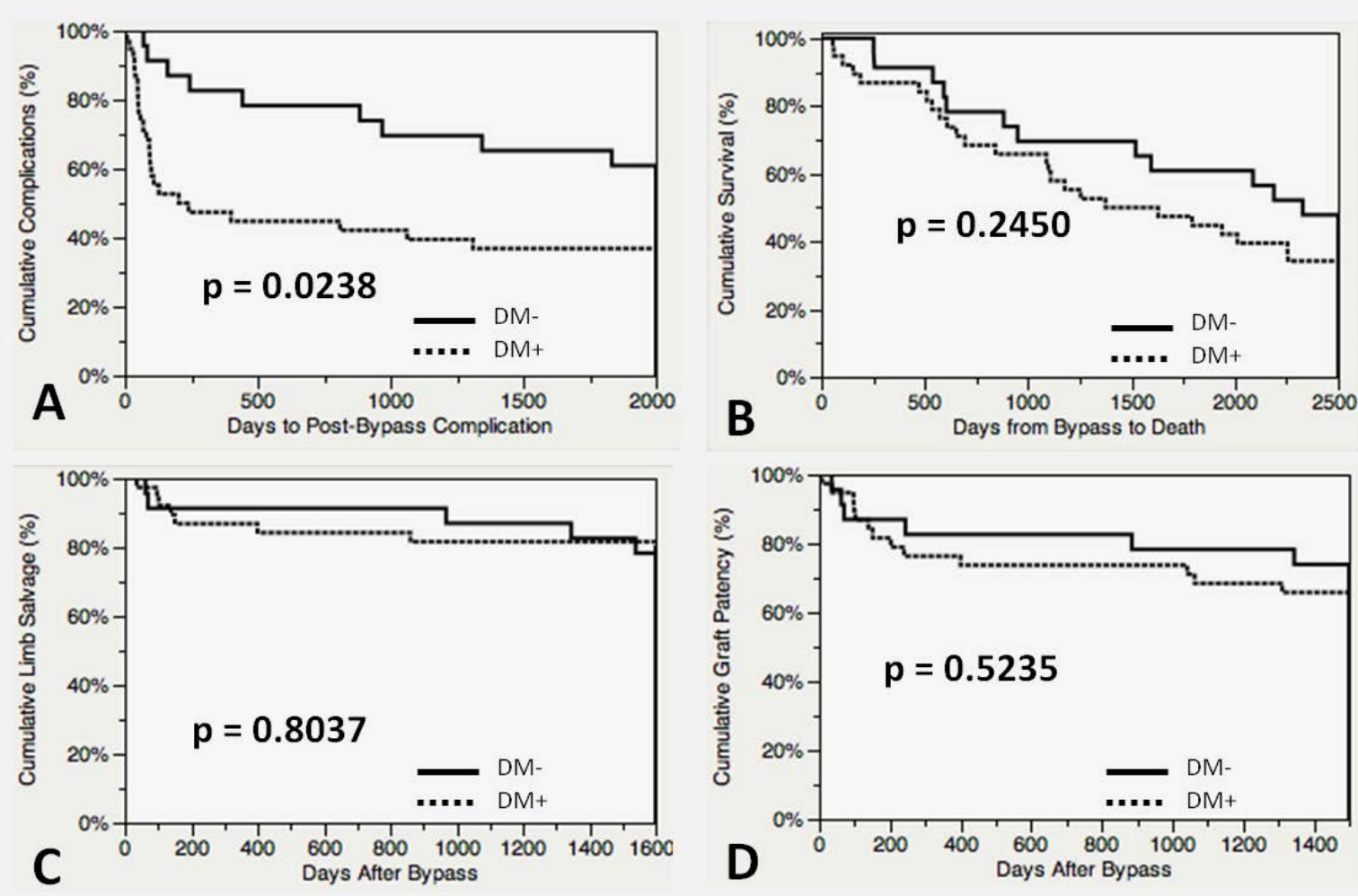

Figure 4

Kaplan - Meier survival curves of patients with diabetes $\left(\mathrm{DM}^{+}\right)$and without diabetes $\left(\mathrm{DM}^{-}\right)$. Cumulative complication rate $(\mathrm{A})$ and survival (B) includes only patients with critical limb ischemia and no bypass failures. In order to analyze cumulative limb salvage (C) and graft patency (D), patients with critical limb ischemia and post-operative bypass failure were included in the analysis. 
Table 3 Post-Operative Outcomes of patients with diabetes $\left(\mathrm{DM}^{+}\right)$and without diabetes $\left(\mathrm{DM}^{-}\right)$with critical limb ischemia who underwent infrainguinal revascularization without graft failure. ${ }^{*}=p<0.05$.

\begin{tabular}{|c|c|c|c|}
\hline & DM- & $\mathbf{D M}^{+}$ & \\
\hline & $(n=16)$ & $(n=27)$ & P-value \\
\hline Cellulitis / Edema & $7(44 \%)$ & $9(33 \%)$ & 0.4946 \\
\hline Dry Gangrene & $1(6 \%)$ & $3(11 \%)$ & 0.5958 \\
\hline Split - Thickness Skin Graft & $0(0 \%)$ & $2(7 \%)$ & 0.2649 \\
\hline Cardiovascular Complication & $1(6 \%)$ & $1(4 \%)$ & 0.7015 \\
\hline Occlusion (Stenosis vs. Thrombosis) & $4(25 \%)$ & $3(11 \%)$ & 0.2331 \\
\hline Claudication & $3(19 \%)$ & $0(0 \%)$ & $0.0197^{*}$ \\
\hline Ulcers & $3(19 \%)$ & $2(7 \%)$ & 0.2621 \\
\hline Retroperitoneal Hematoma & $1(6 \%)$ & $0(0 \%)$ & 0.1887 \\
\hline Angioplasty/Thrombectomy & $3(19 \%)$ & $2(7 \%)$ & 0.2621 \\
\hline Toe/Transmetatarsal Amputation & $3(19 \%)$ & $12(44 \%)$ & 0.0875 \\
\hline Osteomyelitis & $1(6 \%)$ & $3(11 \%)$ & 0.5958 \\
\hline Lymph Leak & $1(6 \%)$ & $2(7 \%)$ & 0.8855 \\
\hline
\end{tabular}

$\mathrm{CLI}$ and chronic intermittent claudication at baseline. 10 patients undergoing infra-inguinal bypass or amputation, monocyte activation remained unchanged [10]. Of the 30 patients in the CLI group, 10 (33\%) had diabetes however diabetics and nondiabetics were analyzed together in the CLI group [10]. Subgroup analysis of this study may have revealed a difference in monocyte activation between patients with and without diabetes. Surgical stress, which is amplified in diabetics [14], can induce inflammation. To eliminate this potentially confounding condition, we chose to record monocyte levels several months after revascularization rather than in the peri-operative period.

Although diabetes and smoking remain the two greatest risk factors for PAD, PAD also increases with age, whereby patients of an age greater than 70 y have a PAD prevalence of $20 \%[15,16]$. In the current study of patients with $\mathrm{CLI}, \mathrm{DM}^{+}$were older than $\mathrm{DM}^{-}$patients, which may be attributed to the greater prevalence of active smoking among non-diabetic patients, leading to earlier onset of PAD. In $\mathrm{DM}^{-}$patients, $87 \%$ were active smokers at the time of bypass and all of the $\mathrm{DM}^{-}$patients who developed graft failure were smokers, whereas there were significantly fewer active smokers in $\mathrm{DM}^{+}$patients. These results conform to the findings of a report involving 16,534 infra-inguinal bypass procedures, in which patients requiring bypass who were non-smokers were more likely to be diabetic than patients who were smokers $(P<0.001)$ [17]. Furthermore, in that study [17], the odds of smokers having early graft failure was $21 \%$ greater than non-smokers ( $p=0.03$ ) [17]. In our study, the comparable rates of graft failure between $\mathrm{DM}^{+}$and $\mathrm{DM}^{-}$ may reflect the increased smoking prevalence in the non-diabetic group. Compared with age- and sex-matched non-smokers, smokers have an increase in monocyte surface expression of CD40 and an increase in platelet monocyte aggregates [18]. Likewise, diabetics have an increase in platelet-monocyte aggregates and soluble CD40L [12], suggesting smoking and diabetes may have similar effects in the pathogenesis of PAD.
Injured endothelium recruits monocytes which then differentiate into macrophages $[4,9]$, which can further be differentiated into pro-inflammatory M1 macrophages or anti-inflammatory M2 macrophages depending on the respective signals received from the microenvironment [15]. The presence, number, and type of macrophages as well as the $M 1 / M 2$ ratio may be more predictive of the development of restenosis or persistent ischemia than the absolute monocyte count $[9,19]$.

In this study, patients with diabetes demonstrated persistent elevation of monocyte levels after vascular bypass as compared with non-diabetic patients. Given the role of monocytes in atherosclerosis and restenosis, elevated monocyte levels after revascularization would theoretically predict the risk for bypass failure in patients; however absolute monocyte counts may not be a reliable marker in patients with diabetes. These hypothesisgenerating findings indicate that future prospective studies are warranted, with characterization of monocyte subtypes and M1/ M2 index, which may provide useful markers for graft failure in patients with diabetes.

\section{Limitations}

The small sample size and retrospective nature of our study limits the impact of its findings. Although a prospective study with increased power would be ideal, a previous study comparing monocyte levels in patients with and without CLI has been performed as a restrospective study of just 32 patients [2], demonstrating that the current study is not without precedent. Potential confounding factors could have been introduced because leukocyte levels were not performed at uniform intervals but instead were drawn within a range of days (pre-operatively) and months (post-operatively). Although patients requiring bypass revision, below/above - knee amputation, or care for persistent CLI symptoms were eliminated from the study, drawing an unscheduled leukocyte level suggests the patient may have been evaluated for an acute inflammatory process. Although this methodology may present a selection bias, it applies to both groups of patients. Like many studies within the VA system, the study population consisted of all men, and there was also little racial diversity, therefore limiting the results in generalized applicability.

One treatment arm that could have been included in this study and may have impacted our results is peripheral angioplasty. The technical feasibility and value of peripheral angioplasty in the treatment of diabetic foot [20] and critical limb ischemia [21] has been well-described. In a meta-analysis of patients undergoing peripheral angioplasty versus bypass, patients who underwent bypass were found to have higher 30 day mortality rates as compared with peripheral angioplasty, however this difference was not observed at 1, 3 and 5 y post-procedure [22]. Furthermore there was no difference in limb salvage rates or amputation-free survival between groups [22]. Thus, the less invasive approach afforded by peripheral angioplasty with similar outcomes as compared with bypass may have impacted the degree of monocyte (de)recruitment in this study.

Macrophages are multi-potent cells that can change their 
phenotype in response to the surrounding microenvironment [9]. Our study did not characterize the different monocyte subtypes nor did it quantify the CD40 concentration. Validating the findings of this study warrants a larger, prospective trial which quantifies the different monocyte subtypes. If the pro-inflammatory to antiinflammatory macrophage ratio emerges as a predictor of active or impending ischemia, this easily obtainable marker could help clinicians take preemptive measures prior to bypass graft failure.

\section{Conclusion}

Both diabetic and non-diabetic patients with critical limb ischemia had similar monocyte levels prior to vascular bypass. After successful surgical revascularization, monocyte levels decreased in non-diabetic patients, but significantly increased in diabetic patients. We hypothesize that the mechanism for this monocyte elevation is due to chronic sub-clinical inflammation and monocyte activation that characterizes diabetes. A larger, prospective trial which includes patients with and without $\mathrm{CLI}$ and characterizes macrophage sub-type predominance may provide insight into the relationship between monocyte levels, diabetes, and bypass graft outcome.

\section{Acknowledgement}

This work was not financially supported. The authors wish to thank Ewa Jaruga - Killeen for her assistance in statistical analysis in the original abstract. 


\section{References}

1 Nasir K, Guallar E, Navas Acien A, Criqui MH, Lima JA (2005) Relationship of monocyte count and peripheral arterial disease: results from the National Health and Nutrition Examination Survey 1999-2002. Arteriosclerosis, thrombosis, and vascular biology 25: 1966-1971.

2 Magri D, Vasilas P, Muto A (2011) Elevated monocytes in patients with critical limb ischemia diminish after bypass surgery. The Journal of surgical research 167: 140-150.

3 Libby P, Plutzky J (2002) Diabetic macrovascular disease: the glucose paradox. Circulation 106: 2760-2763.

4 Seidler RW, Lenter MC, Guth BD, Doods H (2007) Short - term intra - arterial infusion of monocyte chemoattractant protein-1 results in sustained collateral artery growth. Journal of cardiovascular pharmacology and therapeutics 12: 61-68.

5 Beckman JA, Creager MA, Libby P (2002) Diabetes and atherosclerosis: epidemiology, pathophysiology, and management. JAMA: the journal of the American Medical Association 287: 2570-2581.

6 Novo S (2002) Classification, epidemiology, risk factors and natural history of peripheral arterial disease. Diabetes, obesity \& metabolism 4: S1-6.

7 Pentecost MJ, Criqui MH, Dorros G (2003) Guidelines for peripheral percutaneous transluminal angioplasty of the abdominal aorta and lower extremity vessels. Journal of vascular and interventional radiology: JVIR 14: S495-515.

8 Rodrigues SF, Granger DN (2010) Role of blood cells in ischaemiareperfusion induced endothelial barrier failure. Cardiovascular research 87: 291-299.

9 Ostriker A, Horita HN, Poczobutt J, Weiser Evans MC, Nemenoff RA (2014) Vascular smooth muscle cell- derived transforming growth factor - beta promotes maturation of activated, neo-intima lesionlike macrophages. Arteriosclerosis, thrombosis, andvascular biology 34: 877-886.

10 Burdess A, Nimmo AF, Campbell N (2010) Perioperative platelet and monocyte activation in patients with critical limb ischemia. Journal of vascular surgery 52: 697-703.
11 Freedman JE, Loscalzo J (2002) Platelet-monocyte aggregates: bridging thrombosis and inflammation. Circulation 105: 2130-2132.

12 Harding SA, Sommerfield AJ, Sarma J (2004) Increased CD40 ligand and platelet-monocyte aggregates in patients with type 1 diabetes mellitus. Atherosclerosis 176: 321-325.

13 Varo N, Vicent D, Libby $P$ (2003) Elevated plasma levels of the atherogenic mediator soluble CD40 ligand in diabetic patients: a novel target of thiazolidinediones. Circulation 107: 2664-2669.

14 Lin E, Gletsu Miller N (2013) Surgical stress induces an amplified inflammatory response in patients with type 2 diabetes. ISRN obesity.

15 Pellegrin M, Bouzourene K, Poitry Yamate C (2014) Experimental peripheral arterial disease: new insights into muscle glucose uptake, macrophage, and T-cell polarization during early and late stages. Physiological reports. 2: e00234.

16 Poredos P, Jezovnik MK, Kalodiki E (2014) Medical management of patients with peripheral arterial disease. International angiology: a journal of the International Union of Angiology.

17 Selvarajah S, Black JH, Malas MB, Lum YW, Propper BW, et al. (2014) Preoperative smoking is associated with early graft failure after infrainguinal bypass surgery. Journal of vascular surgery 59: 13081314.

18 Harding SA, Sarma J, Josephs DH (2004) Upregulation of the CD40/ CD40 ligand dyad and platelet-monocyte aggregation in cigarette smokers. Circulation 109: 1926-1929.

19 Moreno PR, Bernardi VH, Lopez Cuellar J (1996) Macrophage infiltration predicts restenosis after coronary intervention in patients with unstable angina. Circulation 94: 3098-3102.

20 Ciccone MM, Marchese A, Generali A (2012) Interventional therapy in diabetic foot: risk factors, clinical events and prognosis at one year follow-up (a study of 103 cases). Pakistan journal of biological sciences 15: 789-794.

21 Faglia E, Clerici G, Clerissi J (2009) Long-term prognosis of diabetic patients with critical limb ischemia: a population-based cohort study. Diabetes care 32: 822-827.

22 Fu X, Zhang Z, Liang K (2015) Angioplasty versus bypass surgery in patients with critical limb ischemia-a meta-analysis. International journal of clinical and experimental medicine 8: 10595-10602. 